\title{
Nonlocal Coherent Perfect Absorption
}

\author{
John Jeffers (1) \\ Department of Physics, University of Strathclyde, John Anderson Building, 107 Rottenrow, Glasgow G4 ONG, United Kingdom
}

(Received 13 June 2019; published 1 October 2019)

\begin{abstract}
Coherent absorption that occurs at two spatially separated, macroscopic lossy beam splitters is described. A superposition mode of any phase can be chosen as the absorbed or transparent mode. For a nonclassical two-photon NOON-state input, a superposition of two photons entering via one of two separate input modes, a single photon can survive the pair of beam splitters with certainty, implying nonlocal absorption of a single photon, which can be detected via the interference in the two-photon survival probability.
\end{abstract}

DOI: 10.1103/PhysRevLett.123.143602

Loss in optics is typically thought of as a purely local absorption of light energy, but this does not always give the full picture. At the quantum level entangled states of light can display nonlocal dispersion cancellation or enhancement in dielectric media [1,2] and these effects carry over to the frequency-dependence of the imaginary part of the refractive index $[3,4]$.

The beam splitter is the central element in optical interference experiments. In particular a 50/50 device can be used to demonstrate a striking quantum effect, two-photon interference $[5,6]$, in which two independent identical single photons incident from different ports must exit the beam splitter from the same port. If the beam splitter is lossy it has input-output relations [7]

$$
\begin{aligned}
& \hat{a}_{1 \text { out }}=t \hat{a}_{1 \text { in }}+r \hat{a}_{2 \text { in }}+l \hat{f}_{1} \\
& \hat{a}_{2 \text { out }}=t \hat{a}_{2 \text { in }}+r \hat{a}_{1 \text { in }}+l \hat{f}_{2},
\end{aligned}
$$

with transmission and reflection coefficients $t$ and $r$, loss factor $l=\sqrt{1-|t|^{2}-|r|^{2}}$, and two bosonic noise annihilation operators, $\hat{f}_{1,2}$ that pertain to input noise channels. These noise operators are necessary to ensure that the output operators are mutually independent and satisfy the standard commutation relations. They must be independent of the optical inputs and satisfy $\left[\hat{f}_{1}, \hat{f}_{1}^{\dagger}\right]=\left[\hat{f}_{2}, \hat{f}_{2}^{\dagger}\right]=1$ and $\left[\hat{f}_{1}, \hat{f}_{2}^{\dagger}\right]=-\left(t r^{*}+r t^{*}\right) /\left(1-|t|^{2}-|r|^{2}\right)$. For lossy beam splitter inputs excited with two identical single photons apparent nonlinear absorption can take place, in which the survival rates for one and two photons do not follow the expected distribution based on random deletion of photons [7].

The underlying explanation for this effect came later [8]: the two unmixed superposition modes of the beam splitter inputs do not suffer the same loss. The apparent nonlinear absorption occurs because the input state can be decomposed into two-photon excitations of the two different superposition modes. One of these modes is absorbed and the other is not. This led to a further prediction-that a $50 \%$ absorbing beam splitter could be rendered perfectly absorbing or perfectly transparent depending on the input superposition mode selected [8]. At 50\% absorption $(t=-r=1 / 2, l=1 / \sqrt{2})$ both of the output arm modes contain just one of the superposition modes,

$$
\hat{d}=\frac{1}{\sqrt{2}}\left(\hat{a}_{2}-\hat{a}_{1}\right),
$$

which is passed without loss. The orthogonal mode

$$
\hat{c}=\frac{1}{\sqrt{2}}\left(\hat{a}_{1}+\hat{a}_{2}\right)
$$

is fully absorbed. By interferometrically cycling the input light between these modes the beam splitter can be rendered fully lossy or fully transparent. If only one of the set of input modes 1 or 2 is excited the output exhibits a loss of 50\%. These ideas provided the first inklings that in quantum optics loss is not always describable as a random probabilistic deletion. Whether or not a photon is absorbed by the lossy device can depend on its interferometric mode and the particular absorption of that mode. Classical light follows the same pattern as quantum and can be fully absorbed or not at all.

A simple theory dictates that to observe the effect fully the transmission and reflection coefficients must satisfy $t= \pm r=1 / 2$-phase relations that are proscribed for a lossless device [8]. General consideration of absorbing slabs of material in the thin, high refractive index limit (see Appendix A in [7]) show that for normal incidence of transversely polarized light

$$
t=\frac{1}{1-i \mu}, \quad r=\frac{i \mu}{1-i \mu},
$$




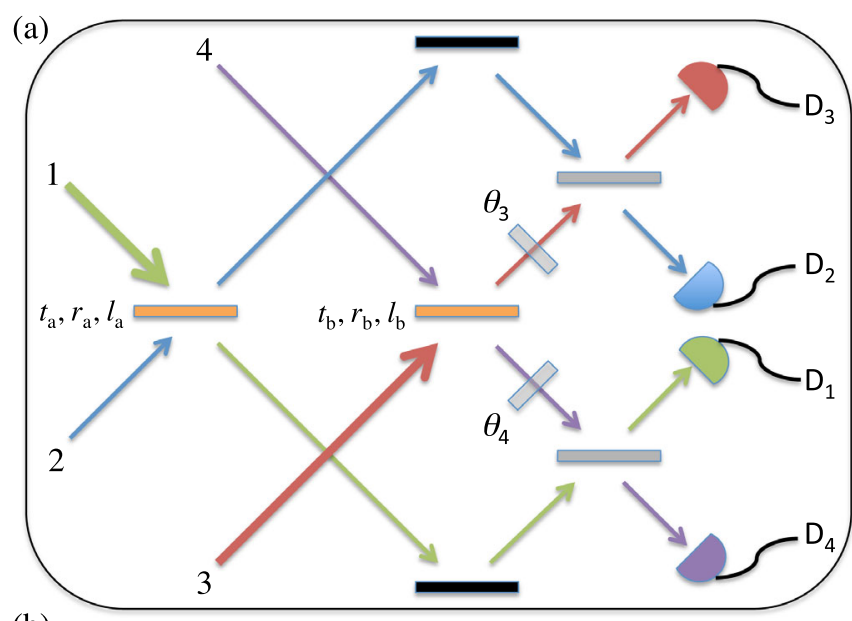

(b)

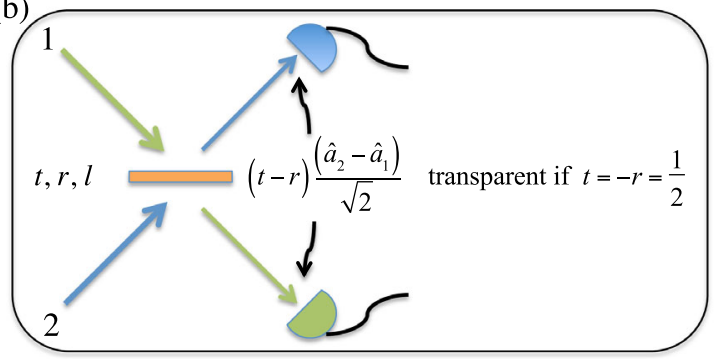

FIG. 1. (a) Setup for two beam splitter nonlocal coherent absorption. Light enters in modes 1 (green) and 3 (red) and impinges on two symmetric $50 \%$ lossy beam splitters a and b. Input modes 2 (blue) and 4 (purple) are in vacuum states. The light is detected interferometrically at four detectors. (b) Standard coherent absorption in which light enters a 50\% lossy beam splitter and, if it enters in the correct superposition of modes 1 and 2 , suffers no absorption.

to first order in $\mu=\omega L n^{2} / c$, where $n=\eta+i \kappa$ is the complex refractive index of the material, of thickness $2 L$. These forms enforce $t=1+r$. For a critically damped medium $(\eta=\kappa) \mu=i \mu_{i}$ is purely imaginary and for $\mu_{i}=1$ the required $50 \%$ absorption is attained. The value of $\mu$ obtained for a finite thickness slab requires a detailed model [9] but for high $n$ it is possible to find a subwavelength thickness for which the absorption is $50 \%$ and which satisfies $t=-r=-1 / 2$. Moreover, significant deviations from $\eta=\kappa$ only give small differences from $50 \%$ absorption.

The first proposal [10] and implementation of means to achieve [11] what has become known as coherent perfect absorption (CPA) provided a physical realization of the tailoring of loss via mode selection and this was extended to subwavelength highly lossy, high refractive index films with plasmonic loss channels - the "lossless lossy" beam splitter [12]. Subsequently the effect was seen with single photons [13], showing that absorption is not necessarily merely probabilistic photon deletion. Two-photon input followed [14], fully verifying the original nonlinear absorption explained in [8]. Some of these effects extend to asymmetric lossy beam splitters [15].
In this Letter the span of CPA effects is extended to encompass nonlocal absorption at separated devices. Previously nonlocal control of CPA at a single absorbing device via polarization has been demonstrated [16]. Here a form of CPA based on loss at more than one absorber is described. In order to illustrate this, two lossy beam splitters are required [Fig. 1(a)]. The setup has four inputs entering via two $50 \%$ lossy beam splitters and four outputs that are overlapped in pairs at another two identical beam splitters whose outputs are monitored at four detectors. The modes, as shown in the Fig. 1(a), have internal paths with an adjustable phase. Only input modes 1 and 3 (of different beam splitters) are excited. All of the other optical modes and all of the input loss modes internal to the beam splitters are unexcited. The comparison with a standard setup is also shown (b), in which CPA occurs because at $50 \%$ absorption both of the output modes contain just one of the superposition modes.

In order to see the coherent effect the light in both the upper and lower halves of Fig. 1(a) must be detected such that it can not give information about either its origin or its path via whichever lossy beam splitter. The detection beam splitter and detectors allow this information to be erased by appropriate adjustment of the phase shifter in each halfspace. This criterion is satisfied if the detectors in modes 1 and 4 (or 2 and 3) detect the same superposition of input modes 1 and 3. A quantum analysis will be used, as in [7,8] so that both classical and quantum effects can be seen. The two output modes that reach the detectors are

$$
\begin{aligned}
& \hat{a}_{1 \text { out }}=t\left(t_{\mathrm{a}} \hat{a}_{1 \text { in }}+r_{\mathrm{a}} \hat{a}_{2 \text { in }}+l_{\mathrm{a}} \hat{f}_{1 \mathrm{a}}\right) \\
& +r e^{i \theta_{4}}\left(t_{\mathrm{b}} \hat{a}_{4 \text { in }}+r_{\mathrm{b}} \hat{a}_{3 \text { in }}+l_{\mathrm{b}} \hat{f}_{4 \mathrm{~b}}\right)+l \hat{f}_{1} \text {, } \\
& \hat{a}_{4 \text { out }}=t e^{i \theta_{4}}\left(t_{\mathrm{b}} \hat{a}_{4 \text { in }}+r_{\mathrm{b}} \hat{a}_{3 \text { in }}+l_{\mathrm{b}} \hat{f}_{4 \mathrm{~b}}\right) \\
& +r\left(t_{\mathrm{a}} \hat{a}_{1 \text { in }}+r_{\mathrm{a}} \hat{a}_{2 \text { in }}+l_{\mathrm{a}} \hat{f}_{1 \mathrm{a}}\right)+l \hat{f}_{4},
\end{aligned}
$$

where, for example, $t_{\mathrm{a}}, r_{\mathrm{a}}, l_{\mathrm{a}}$, and $\hat{f}_{1 \mathrm{a}}$ are, respectively, the transmission, reflection, loss coefficients, and one of the loss mode bosonic operators of beam splitter a, $\theta_{4}$ is an interferometric phase difference between paths 1 and 4 , and $\hat{f}_{1}$ and $\hat{f}_{4}$ are two bosonic loss mode operators of the detection beam splitter (if required). Beam splitters $a$ and $b$ are assumed to be identical and satisfy $t_{\mathrm{a}, \mathrm{b}}=-r_{\mathrm{a}, \mathrm{b}}=1 / 2$, only differing in that they have independent noise modes.

If the irrelevant unexcited optical modes and possible loss modes associated with the detection beam splitter are ignored, the output operators are

$\hat{a}_{1 \text { out }} \sim t t_{\mathrm{a}} \hat{a}_{1 \text { in }}+r e^{i \theta_{4}} r_{\mathrm{b}} \hat{a}_{3 \text { in }}+t l_{\mathrm{a}} \hat{f}_{1 \mathrm{a}}+r e^{i \theta_{4}} l_{\mathrm{b}} \hat{f}_{4 \mathrm{~b}}$,

$\hat{a}_{4 \text { out }} \sim t e^{i \theta_{4}} r_{\mathrm{b}} \hat{a}_{3 \text { in }}+r t_{\mathrm{a}} \hat{a}_{1 \text { in }}+t e^{i \theta_{4}} l_{\mathrm{b}} \hat{f}_{4 \mathrm{~b}}+r l_{\mathrm{a}} \hat{f}_{1 \mathrm{a}}$.

The optical part of these two output modes must be proportional to the same input superposition, which imposes the requirement on the detection beam splitter 
that $t= \pm r$, which is possible only if the detection beam splitter is itself $50 \%$ lossy. This is equivalent to limiting the detection efficiency to $50 \%$, but changes nothing material. Indeed it is possible to circumvent this requirement in other experimental geometries, such as a Young's slits arrangement with the slits replaced by coherently absorbing beam splitters. The output operators are

$$
\begin{aligned}
\hat{a}_{1 \text { out }} & \sim \frac{1}{2}\left(t_{\mathrm{a}} \hat{a}_{1 \text { in }}-e^{i \theta_{4}} r_{\mathrm{b}} \hat{a}_{3 \text { in }}+l_{\mathrm{a}} \hat{f}_{1 \mathrm{a}}-e^{i \theta_{4}} l_{\mathrm{b}} \hat{f}_{4 \mathrm{~b}}\right) \\
& =\frac{1}{2}\left(\frac{1}{2}\left(\hat{a}_{1 \text { in }}+e^{i \theta_{4}} \hat{a}_{3 \text { in }}\right)+\frac{1}{\sqrt{2}}\left(\hat{f}_{1 \mathrm{a}}-e^{i \theta_{4}} \hat{f}_{4 \mathrm{~b}}\right)\right) \\
& \sim-\hat{a}_{4 \text { out }} .
\end{aligned}
$$

The first line of Eq. (7) shows that the output operators in modes 1 and 4 depend upon the input operators in modes 1 and 3 weighted by transmission and reflection coefficients at different beam splitters. The loss mode operator is a sum of two loss-mode operators for propagation into mode 1 at beam splitter a and mode 4 at beam splitter $b$, weighted by the overall loss at the relevant device. In the second line standard 50\% CPA relations between transmission and reflection coefficients are assumed, showing that both output operators then depend upon the same superposition of input optical operators and loss-mode operators. The output operators cycle between the input superposition operators,

$$
\begin{aligned}
& \hat{c}_{13}=\frac{1}{\sqrt{2}}\left(\hat{a}_{1}+\hat{a}_{3}\right), \\
& \hat{d}_{13}=\frac{1}{\sqrt{2}}\left(\hat{a}_{3}-\hat{a}_{1}\right),
\end{aligned}
$$

depending on the internal phase $\theta_{4}$. A similar analysis to that from Eq. (5) onwards, but applied to detection modes 2 and 3 gives

$$
\begin{aligned}
\hat{a}_{3 \text { out }} & \sim \frac{1}{2}\left(\frac{1}{2}\left(\hat{a}_{1 \text { in }}+e^{i \theta_{3}} \hat{a}_{3 \text { in }}\right)+\frac{1}{\sqrt{2}}\left(\hat{f}_{1 \mathrm{a}}-e^{i \theta_{3}} \hat{f}_{4 \mathrm{~b}}\right)\right) \\
& \sim-\hat{a}_{2 \text { out }},
\end{aligned}
$$

so if the two phases are the same then all four detectors detect excitations of the same superposition of the two separate inputs to different beam splitters. The orthogonal mode $\frac{1}{2}\left(\hat{a}_{1 \text { in }}-e^{i \theta_{3}} \hat{a}_{3 \text { in }}\right)$ is not output to the detectors. Light that excites this mode is completely absorbed jointly by the two lossy beam splitters. By cycling the input between these two superpositions the device can be made entirely transparent or completely absorbing-the signature of coherent absorption (disregarding the 50\% maximum detection efficiency). As in the single splitter version of the effect, if only one of the input modes at one beam splitter is excited the light suffers a 50\% loss before reaching the detection beam splitters. Here only one mode of each of the input beam splitters is excited so CPA cannot occur locally at either of these devices.

One aspect of this two-beam splitter coherent absorption is that, provided that the detectors provide no information about which beam splitter the light passed, superpositions of the input modes of any phase can pass the compound device as if it were transparent. The orthogonal superposition is always fully absorbed. This is more general than single beam splitter coherent absorption, which always passes the d-mode superposition [Eq. (2)] and absorbs the c-mode superposition [Eq. (3)]. Furthermore, other spatial input mode superpositions can exhibit coherent absorption-modes 1 and 3 , modes 1 and 4 , modes 2 and 3 , and modes 2 and 4 . The coefficient $t_{\mathrm{a}}$ or $r_{\mathrm{a}}$ can be coherently added to either $t_{\mathrm{b}}$ or $r_{\mathrm{b}}$. Neither is the effect restricted to a pair of beam splitters. It can occur for any set of input 50\% absorbing splitters if the output modes are all proportional to the same relevant interferometric sum of excited input modes.

The coherent absorption effect described above is nonlocal in that the overall survival coefficients of the absorbed and nonabsorbed modes depend on oppositely phased sums of two transmission and/or reflection coefficients at two different, spatially separated macroscopic beam splitters. There is, however, nothing specifically nonlocal in the quantum sense about the effect. It works for any state of the relevant superposition modes. This is exactly as for standard CPA. This nonlocality can be exploited to investigate what happens in a quantum case by using as input a pair of photons in the state

$$
\left|2_{\phi}\right\rangle=\frac{1}{\sqrt{2}}\left[|2,0,0,0\rangle+e^{i \phi}|0,0,2,0\rangle\right],
$$

where the first quantum number is the number of photons in input mode 1 etc. This so-called NOON state of two photons in either mode 1 or 3 corresponds to the output state of the coalescence beam splitter in a standard two-photon interference experiment $[5,6]$.

The state can be rewritten in terms of the input superposition operators [Eq. (8)] as

$$
\begin{aligned}
\left|2_{\phi}\right\rangle & =\frac{1}{4}\left[\left(\hat{c}_{13}^{\dagger}-\hat{d}_{13}^{\dagger}\right)^{2}+e^{i \phi}\left(\hat{c}_{13}^{\dagger}+\hat{d}_{13}^{\dagger}\right)^{2}\right]|\{0\}\rangle \\
& =\frac{1}{2}\left[\hat{c}_{13}^{\dagger 2}+\hat{d}_{13}^{\dagger}\right]|\{0\}\rangle \quad \text { for } \phi=0 \\
& =\hat{c}_{13}^{\dagger} \hat{d}_{13}^{\dagger}|\{0\}\rangle \quad \text { for } \phi=\pi,
\end{aligned}
$$

which for $\phi=0$ is a superposition of two photons in either superposition mode and for $\phi=\pi$ is a state with one photon in each superposition mode. If the phases are set appropriately, i.e., $\theta_{4}=\theta_{3}=0, \pi$ then for $\phi=0$ there is an enhanced probability of two-photon survival of 0.5 . For $\phi=\pi$ exactly one photon survives to reach the detection beam splitters. This is exactly the nonlinear absorption 


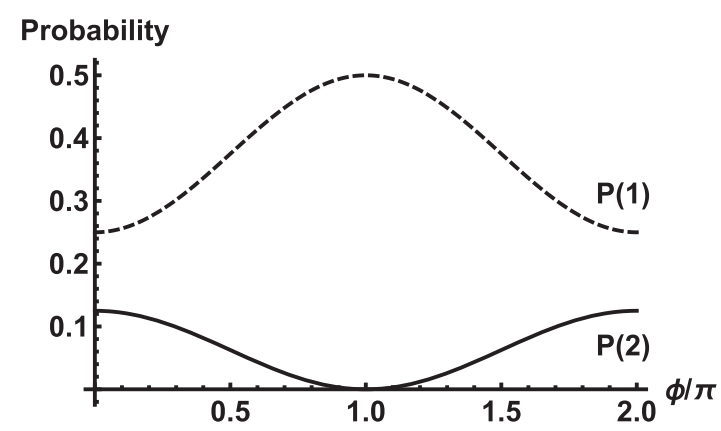

FIG. 2. Plot of the one- and two-count detection probability against input state superposition phase for photon number discriminating, perfect quantum efficiency detectors in each output arm.

scenario first described in $[7,8]$ for one lossy beam splitter. A plot of the one- and two-photon survival probabilities as a function of $\phi$ shows (in principle) unit visibility oscillations, but the one-photon and two-photon probabilities are out of phase. In practice (Fig. 2) the 50\% maximum detection efficiency lowers the maximum survival probabilities (at any detector) and limits the one-count visibility to $33 \%$. The two-count visibility is unaffected-if two photons do not survive beam splitters a and $b$ two photons can never be detected. Similarly, this visibility is unaffected if detectors do not discriminate between different photon numbers, or if they suffer from nonunit quantum efficiency.

If the phases are adjusted such that exactly one photon survives, i.e., at a zero in the two-count probability in Fig. 2, one can ask the simple question "Where is the photon absorbed?" One might guess that it is absorbed either at beam splitter a or beam splitter b with equal, $50 \%$ probability. The guess is correct, but the answer is more surprising. Again, take as input state Eq. (10) with $\phi=\pi$ so that there is one photon in each superposition mode. The internal phases are set at $\theta_{3}=\theta_{4}=0$ and all of the internal loss channel inputs are assumed to be in their ground states. Then the inverse relations for the lossy beam splitters can be used to provide the multimode output state, including loss channel states. By restricting this to situations where one photon is lost at beam splitters a or b and none are lost elsewhere all components of the state are traced out except the one that is a product of two one-photon excitations of the modes

$$
\begin{aligned}
\left|2_{\text {out }}\right\rangle= & \frac{1}{2}\left(\hat{a}_{1 \text { out }}^{\dagger}-\hat{a}_{2 \text { out }}^{\dagger}+\hat{a}_{3 \text { out }}^{\dagger}-\hat{a}_{4 \text { out }}^{\dagger}\right) \\
& \times \frac{1}{\sqrt{2}}\left(\hat{g}_{1 \mathrm{a}}^{\dagger}-\hat{g}_{3 \mathrm{~b}}^{\dagger}\right)|\{0\}\rangle,
\end{aligned}
$$

where $\hat{g}_{1 \mathrm{a}}^{\dagger}$ and $\hat{g}_{3 \mathrm{~b}}^{\dagger}$ are output noise mode creation operators for input from mode 1 and 3, respectively. A further trace over either noise mode provides the probability that the photon is lost at either beam splitter. However this is not the only basis. Instead a trace over the nonlocal noise mode orthogonal to the operator in Eq. (12) can be performed. This trace affects the state by reducing the number of noise modes from two, one associated with a loss channel at each beam splitter, to one nonlocal mode of two beam splitters $(1 / \sqrt{2})\left(\hat{g}_{1 \mathrm{a}}^{\dagger}-\hat{g}_{3 \mathrm{~b}}^{\dagger}\right)$, into which the absorbed photon disappears. In effect the single absorption creates the joint state of the optical outputs and beam splitter loss modes

$$
\begin{aligned}
\left|2_{\text {out }}\right\rangle= & \frac{1}{2}(|1000\rangle-|0100\rangle+|0010\rangle-|0001\rangle) \\
& \times \frac{1}{\sqrt{2}}(|10\rangle-|01\rangle),
\end{aligned}
$$

where the first line is the state of the four optical modes and the second is the entangled output state of the noise modes of the beam splitters. The effect is similar to the creation of entangled vibrational states of diamond used for quantum memories [17]. The entanglement here would require an interference experiment between the two loss channels to verify. There have been bosonic interference experiments for some plasmonic systems [18]. In any case the perfect visibility in the two-photon probability in Fig. 2 is an unambiguous signature of perfect nonlocal absorption of a single photon. Experimental interference in the two-photon detection rate at any visibility would be an indication that the effect is occurring.

Coherent absorption is sometimes explained, at least classically, as controlling the loss experienced by a standing-wave field by placing a subwavelength, lossy film at a node or antinode, although this was not a requirement in the original proposal [10]. Here an extended device demonstrating CPA based on sums of reflection and transmission coefficients at different, macroscopic, spatially separated absorbers is described. Coherent absorption can not occur locally so the standard explanation is difficult to apply. Furthermore the effect described is more general, in that a superposition of input modes of any relative phase can undergo CPA- a consequence of the extra freedom provided by the flexibility of the two interferometric phases and that the absorbing device is composed of two lossy beam splitters, with extra modes. This is a different level of control in that not only the loss can be tailored, but coherent absorption itself.

The above also raises a further interesting point. Loss within an interferometer normally degrades interference visibility. Here this does not necessarily happen. Beam splitters are essential components of quantum optical networks and are deployed in simple optical quantum information processors with increasing depth, for example in boson sampling experiments $[19,20]$. All of the beam splitters in such experiments are lossy, but of course never with an absorption close to 50\%. However, coherent absorption effects remain away from 50\% loss [10] and their effect should persist, particularly when there is no 
significant optical propagation phase within individual beam splitters. Also, the effect is not limited to two devices; there is no restriction on the number of beam splitters whose coefficients can be added coherently to exhibit nonlocal coherent absorption. It will occur naturally in both classical and quantum-optical processing schemes and can be controlled and exploited in both. As a simple example of a network, we can extend that in Fig. 1 to include more identical stages with a central beam splitter, similar to the one with two mirrors vertically-aligned with beam splitter $b$. Then the initial nonlocal coherent absorption effect can persist perfectly, propagated to subsequent stages via local coherent absorption.

Finally, a photon can be detected by an absorbing detector in only one place but, as a single-photon version of Taylor's low-intensity interference experiment shows, it can sometimes be detected in a way that suggests that it might have been in two places at once at some earlier time $[21,22]$. However a photon cannot be registered by two spacelike-separated, macroscopic detectors of any kind. In this work, in the quantum regime, for two photon NOONstate input, both photons enter an interferometer through the same beam splitter (although which one is not known a priori). The control of coherent absorption allows precisely one photon to be absorbed with certainty into a single nonlocal loss mode, implementing apparent nonlinear nonlocal absorption. This is a situation where two photons enter the device through the same beam splitter, but one of the two photons is absorbed jointly by two visibly macroscopic beam splitters.

The author would like to thank Steve Barnett for useful comments on this work, which was supported by the UK Engineering and Physical Sciences Research Council through the Quantum Communications Hub Grant, No. EP/M013472/1 and the Quantum Imaging Hub Grant, No. EP/M01326X/1.

[1] A. M. Steinberg, P. G. Kwiat, and R. Y. Chiao, Phys. Rev. Lett. 68, 2421 (1992).

[2] J. D. Franson, Phys. Rev. A 45, 3126 (1992).

[3] J. Jeffers and S. M. Barnett, Phys. Rev. A 47, 3291 (1993).
[4] T. A. Brun and S. M. Barnett, J. Mod. Opt. 45, 777 (1998).

[5] H. Fearn and R. Loudon, Opt. Commun. 64, 485 (1987).

[6] C. K. Hong, Z. Y. Ou, and L. Mandel, Phys. Rev. Lett. 59, 2044 (1987).

[7] S. M. Barnett, J. Jeffers, A. Gatti, and R. Loudon, Phys. Rev. A 57, 2134 (1998).

[8] J. Jeffers, J. Mod. Opt. 47, 1819 (2000).

[9] C. Hägglund, S. P. Aspell, and B. Kasemo, Nano Lett. 10, 3135 (2010).

[10] Y. D. Chong, L. Ge, H. Cao, and A. D. Stone, Phys. Rev. Lett. 105, 053901 (2010).

[11] W. Wan, Y. D. Chong, L. Ge, H. Noh, A. D. Stone, and H. Cao, Science 331, 889 (2011).

[12] J. Zhang, K. F. MacDonald, and N. I. Zheludev, Light 1, e18 (2012).

[13] T. Roger, S. Vezzoli, E. Bolduc, J. Valente, J. J. F. Heitz, J. Jeffers, C. Soci, J. Leach, C. Couteau, N. I. Zheludev, and D. Faccio, Nat. Commun. 6, 7031 (2015).

[14] T. Roger, S. Restuccia, A. Lyons, D. Giovannini, J. Romero, J. Jeffers, M. Padgett, and D. Faccio, Phys. Rev. Lett. 117, 023601 (2016).

[15] R. Uppu, T. A. W. Wolterink, T. B. H. Tentrup, and P. W. H. Pinkse, Opt. Express 24, 16440 (2016).

[16] C. Altuzarra, S. Vezzoli, J. Valente, W. Gao, C. Soci, D. Faccio, and C. Couteau, ACS Photonics 4, 2124 (2017).

[17] I. A. Walmsley, K. C. Lee, M. Sprague, B. Sussman, J. Nunn, N. Langford, X.-M. Jin, T. Champion, P. Michelberger, K. Reim, D. England, and D. Jaksch, J. Phys. Conf. Ser. 442, 012004 (2013).

[18] R. W. Heeres, L. P. Kouwenhoven, and V. Zwiller, Nat. Nanotechnol. 8, 719 (2013).

[19] J. B. Spring, B. J. Metcalf, P. C. Humphreys, W. S. Kolthammer, X.-M. Jin, M. Barbieri, A. Datta, N. Thomas-Peter, N. K. Langford, D. Kundys, J. C. Gates, B. J. Smith, P. G. R. Smith, and I. A. Walmsley, Science 339, 798 (2013).

[20] H. Wang, W. Li, X. Jiang, Y.-M. He, Y.-H. Li, X. Ding, M.-C. Chen, J. Qin, C.-Z. Peng, C. Schneider, M. Kamp, W.-J. Zhang, H. Li, L.-X. You, Z. Wang, J. P. Dowling, S. Höfling, C.-Y. Lu, and J.-W. Pan, Phys. Rev. Lett. 120, 230502 (2018).

[21] P. Grangier, G. Roger, and A. Aspect, Europhys. Lett. 1, 173 (1986).

[22] G. I. Taylor, Proc. Cambridge Philos. Soc. 15, 114 (1909). 